\title{
LIBERAÇÃO BIFÁSICA DE BUTANO EM AMBIENTE ABERTO PARA CLASSIFICAÇÃO DE ÁREA DE RISCO ATRAVÉS DE CFD
}

\author{
P. L. BARROS ${ }^{1}$, T. C. L. OLIVEIRA ${ }^{1}$ e J. J. N. ALVES ${ }^{1}$ \\ ${ }^{1}$ Universidade Federal de Campina Grande, Unidade Acadêmica de Engenharia Química \\ E-mail para contato: paloma.lins@eq.ufcg.edu.br
}

\begin{abstract}
RESUMO - O método de classificação de área de risco surgiu a partir da necessidade da indústria de desenvolver técnicas de segurança adequadas e diminuir a probabilidade de ocorrência de ignição na planta. Com isto, este trabalho tem o objetivo de prever via fluidodinâmica computacional a liberação bifásica em ambiente aberto e determinar a extensão da nuvem de dispersão gasosa para classificação de área. A simulação do caso estudado consiste em uma emissão contínua e atomizada de butano a partir do orifício de liberação. Utilizou-se uma abordagem Euleriana-Euleriana de forma que a fase vapor é contínua e a fase líquida é dispersa, sendo as condições de contorno determinadas a partir de condições específicas de armazenamento. A pluma de gás forma uma zona de expansão e outra de arrastamento, podendo ser observada pelo perfil de fração volumétrica ao longo do eixo de liberação. Foram obtidas a extensão da pluma para o limite inferior de explosividade (LIE) e para a metade deste, de forma a aumentar o nível de segurança. Para o último caso, a extensão mostrou-se aproximadamente duas vezes maior do que o primeiro.
\end{abstract}

\section{INTRODUÇÃO}

A ocorrência de acidentes originados por uso indevido de equipamentos elétricos em áreas passíveis de ocorrer atmosfera explosiva devido à liberação de substâncias inflamáveis acontece em várias partes do mundo, como exposto por Bossert (2001). Equipamentos elétricos são potenciais fontes de ignição, o que pode provocar explosões dentro de uma área industrial quando requisitos de segurança não são incorporados.

A classificação de áreas, portanto, consiste em uma técnica para minimizar a probabilidade de ignição acidental em ambientes de atmosferas explosivas (Eckhoff, 2016). O estudo desta técnica é de muita relevância para o meio industrial e, com isso, as normas internacionais estão sempre sendo atualizadas de acordo com a tecnologia em uso. Em paralelo, algumas indústrias do petróleo, química e da petroquímica podem desenvolver seus próprios padrões aplicáveis às suas condições particulares.

Em alternativa à modelagem matemática utilizada atualmente, tem-se o emprego de modelos rigorosos em CFD para fornecer uma melhor análise e visualização da liberação de gases, líquidos ou misturas bifásicas passíveis de formar atmosferas explosivas. A análise em CFD permite delimitar a extensão da pluma de gás inflamável em áreas classificadas de forma 
otimizada e precisa, possibilitando desta forma o uso de instalações seguras e a diminuição de custos devido ao fato de evitar o superdimensionamento de áreas classificadas.

\subsection{Classificação de área}

Classificação de áreas é a divisão de uma planta industrial em regiões não-perigosas e regiões perigosas. Estas últimas dividem-se em zonas de acordo com a probabilidade de existência e duração da mistura entre gases explosivos e ar atmosférico, que por sua vez depende das propriedades químicas do material inflamável, taxa de liberação, grau de ventilação do ambiente, concentração e outros fatores.

A subdivisão em zonas influencia diretamente os equipamentos e aparatos elétricos utilizados na região, os quais devem ser devidamente selecionados com segurança. Estes equipamentos, específicos e certificados, devem ser corretamente instalados e adequadamente mantidos de acordo com a classificação e com os padrões técnicos requeridos.

As zonas devem ser documentadas em mapas que mostram toda a extensão nas três dimensões, são elas: zona 0 , zona 1 e zona 2 . A zona 0 corresponde à atmosfera explosiva presente continuamente ou por longos períodos. A zona 1 tem uma provável atmosfera explosiva por curtos períodos durante operação normal. E, por fim, a zona 2 possui baixa probabilidade de apresentar atmosfera explosiva durante operação normal, em caso de ocorrência ela se dará somente por curtos períodos de tempo.

Zohdirad et al. (2016) enfatiza que a classificação de áreas subestimadas é considerada como erro e, portanto, devem ser evitadas. Por outro lado, a superestimação pode se tornar bastante onerosa. Nesse ponto encontra-se o desafio de precisão na técnica de classificação de áreas e a importância da aplicação de fluidodinâmica computacional como ferramenta.

\subsection{Formas de liberação}

Muitas substâncias químicas inflamáveis são armazenadas ou transportadas na forma de gases comprimidos, gases liquefeitos ou líquidos. Liberações destas substâncias no ambiente podem acontecer igualmente em diferentes estados termodinâmicos, estando sujeitas às condições de armazenamento, de ambiente e propriedades físicas.

De forma simplificada a Tabela 1, adaptada de TNO Yellow Book (2005), apresenta as regiões do orifício e o tipo de emissão que pode ser determinado para o caso de armazenamento de gases liquefeitos.

Tabela 1 - Identificação qualitativa do tipo de liberação

\begin{tabular}{|c|c|}
\hline Região do orifício & Liberação \\
\hline \hline Acima do nível do líquido & Vapor \\
\hline No nível do líquido & Bifásico \\
\hline Abaixo do nível do líquido & Líquido \\
\hline
\end{tabular}

\section{METODOLOGIA}

A substância inflamável escolhida para realizar a simulação da liberação bifásica foi o butano em regime estacionário. Utilizou-se o modelo de turbulência $k-\varepsilon$ com uma abordagem 
Euleriana-Euleriana cuja a fase vapor é contínua e a fase líquida é dispersa. A configuração de liberação analisada é contínua e forma duas fases a partir do ponto de liberação, sendo $1 \times 10^{-4}$ o valor do critério de convergência da simulação. A determinação do passo de tempo usado foi igual a $1 \times 10^{-5}$ até a $20^{\mathrm{a}}$ iteração e $5 \times 10^{-4}$ para as iterações seguintes.

\subsection{Geometria e malha}

As Figuras 1 e 2 ilustram respectivamente a forma de liberação e as dimensões do sistema simulado no software ANSYS CFX ${ }^{\circledR}$.

Figura 1 - Esquema representativo de liberação bifásica

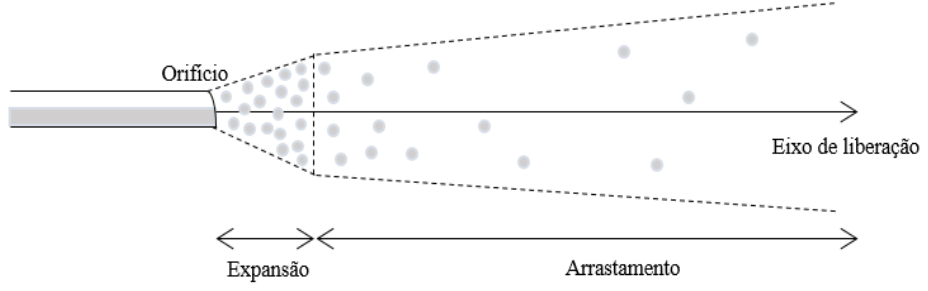

Figura 2 - Representação geométrica em 2D do sistema simulado

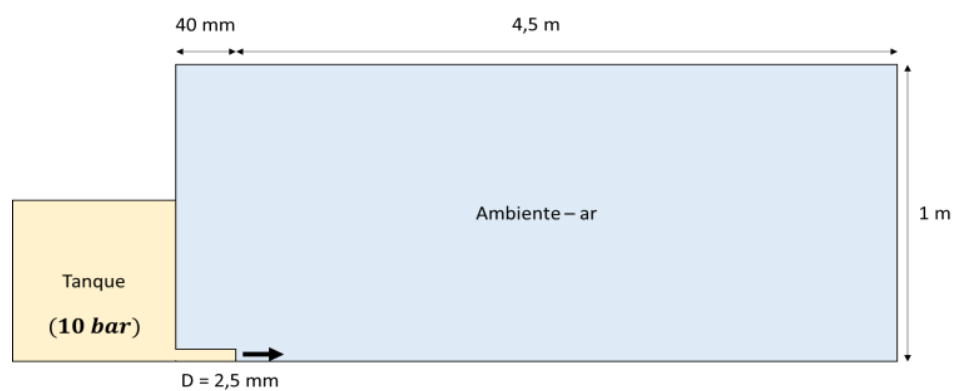

Haja vista que existe simetria na liberação da mistura bifásica, uma fatia de $4^{\circ}$ do sistema foi utilizada para construir a geometria. O domínio utilizado para a simulação consiste no sistema após a saída do orifício e, portanto, não inclui o tanque de armazenamento. A Figura 3 mostra a geometria e a malha de 54040 elementos construída.

Figura 3 - Visão isométrica da geometria e malha gerada

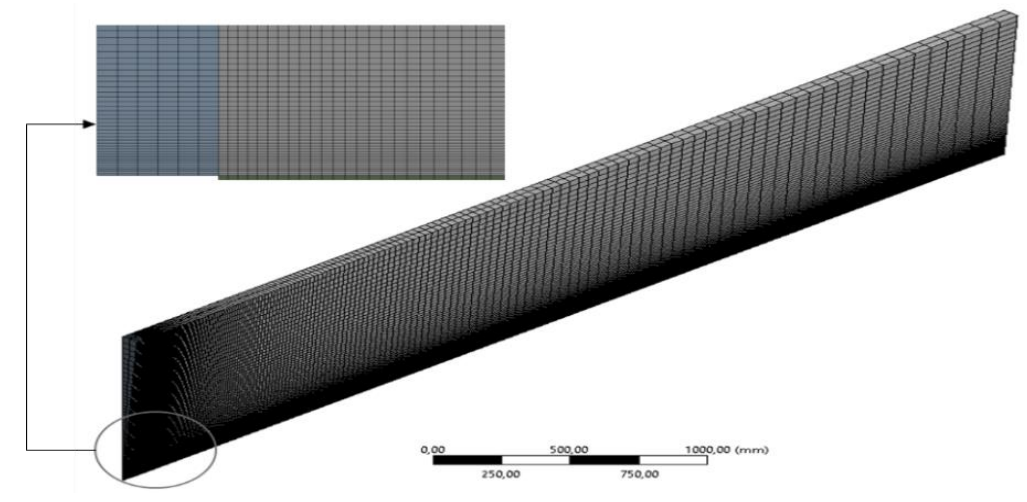

ANSYS

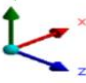




\subsection{Condições de contorno}

A taxa de emissão da mistura inflamável bifásica é avaliada em função das condições de armazenamento e das propriedades da substância. Ela varia entre a velocidade calculada pela equação de Bernoulli e a velocidade do som no gás. De acordo com Sissom e Pitts (1988), o sistema exibirá velocidade sônica se a condição apresentada na Tabela 2 for satisfeita.

Tabela 2 - Escolha de condições de contorno.

\begin{tabular}{|c|c|c|}
\hline Regime & Subsônico & Sônico \\
\hline \hline Condição & $\frac{P_{a m b}}{P_{a r m}}>\left(\frac{2}{\gamma+1}\right)^{\frac{\gamma}{\gamma-1}}$ & $\frac{P_{a m b}}{P_{a r m}} \leq\left(\frac{2}{\gamma+1}\right)^{\frac{\gamma}{\gamma-1}}$ \\
\hline Velocidade (m/s) & $C_{d} \sqrt{\frac{2\left(\mathrm{P}_{\mathrm{arm}}-\mathrm{P}_{\mathrm{amb}}\right)}{\rho_{l}}}$ & $\sqrt{\frac{\gamma R T}{M M}}$ \\
\hline $\begin{array}{c}\text { Temperatura no } \\
\text { orifício (K) }\end{array}$ & $T_{\text {arm }}\left(\frac{P_{a m b}}{P_{\text {arm }}}\right)^{\frac{\gamma-1}{\gamma}}$ & $T_{\text {arm }}\left(\frac{2}{\gamma+1}\right)$ \\
\hline
\end{tabular}

O cálculo da fração mássica vaporizada e diâmetro médio de gota foram realizados como mostrado por Calay e Holdo (2008) nas Equações 3 e 4 respectivamente.

$$
\begin{aligned}
& x_{\text {flash }}^{\text {gas }}=\frac{C p_{l}\left(T_{\text {arm }}-T_{b}\right)}{\Delta H_{\text {vap }}} \\
& d_{32}=0,585 \frac{1}{v} \sqrt{\frac{\sigma}{\rho_{l}}}
\end{aligned}
$$

Para uma pressão de 10 bar no tanque de armazenamento, foram calculadas as condições iniciais apresentadas na Tabela 3.

Tabela 3 - Condições de contorno dos casos simulados.

\section{RESULTADOS}

\begin{tabular}{|c|c|}
\hline Especificação & Valor \\
\hline Diâmetro médio da gota $(\mu \mathrm{m})$ & 11,335 \\
\hline Velocidade de liberação $(\mathrm{m} / \mathrm{s})$ & 229,7462 \\
\hline Fração volumétrica do gás & 0,9989 \\
\hline Fração volumétrica do líquido & 0,0011 \\
\hline Temperatura no orifício $(\mathrm{K})$ & 337,8567 \\
\hline
\end{tabular}

A representação gráfica da pluma e o decaimento da fração molar com a posição foram analisados após a simulação, ambos são apresentados nas Figuras 4 e 5. 
Figura 4 - Fração molar do gás próximo ao orifício

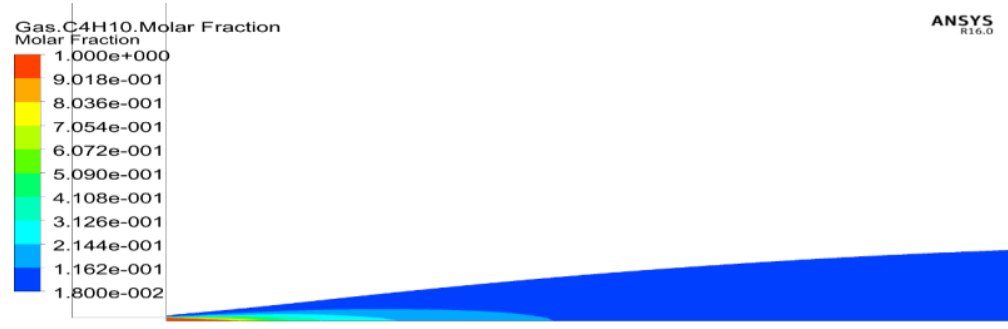

Figura 5 - Decaimento da fração volumétrica de butano

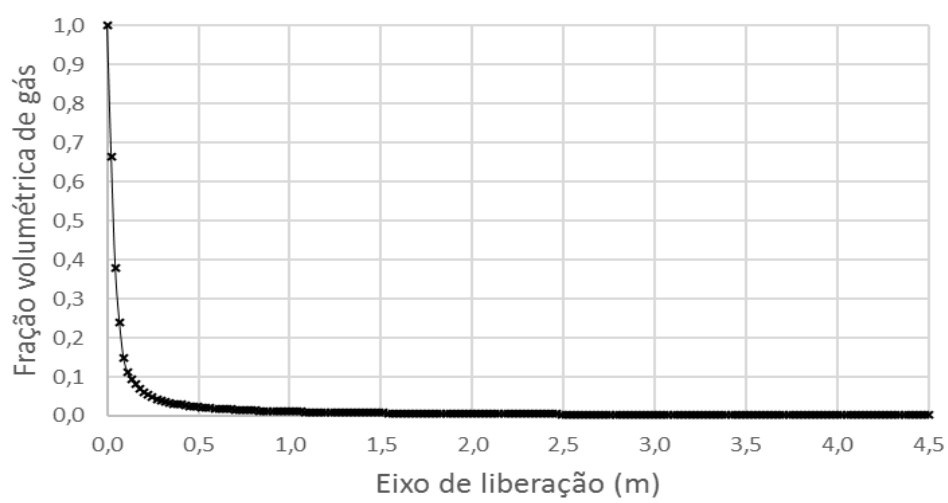

A Tabela 4 apresenta a extensão da pluma gasosa avaliada no limite inferior de explosividade (1,8 vol\%), em $50 \%$ e em $25 \%$ do LIE.

Tabela 4 - Resultados de extensão da pluma.

\begin{tabular}{|c|c|c|}
\hline Extensão LIE $(\mathrm{m})$ & Extensão 1/2 LIE $(\mathrm{m})$ & Extensão 1/4 LIE $(\mathrm{m})$ \\
\hline 0,574 & 1,140 & 2,258 \\
\hline
\end{tabular}

Observa-se que a extensão do gás em $50 \%$ do limite inferior de explosividade (LIE) é aproximadamente duplicada em comparação com a extensão no LIE. De forma semelhante, a extensão novamente dobra para a análise em 1/4 do LIE comparado com o resultado em 1/2 do LIE.

\section{CONCLUSÕES}

A modelagem da emissão bifásica do butano permitiu prever a taxa de liberação, diâmetro de gota e fração volumétrica das fases, parâmetros estes que foram utilizados como condições de contorno na simulação. Os resultados mostraram o perfil de fração molar do butano no plano de liberação e, portanto, foi obtida a extensão da pluma gasosa tanto no limite inferior de explosividade (LIE) quanto na metade deste para uma maior segurança de operação. A extensão da nuvem dispersa de gás é uma das informações desejadas para classificação de áreas de risco e consequente subdivisão da região em zonas, no entanto, é esperado um estudo mais completo que inclua probabilidade de emissão e quantificação do volume da pluma. Para a liberação 
contínua de butano bifásico e atomizado a partir do orifício, a extensão prevista por CFD foi de 0,57 m no LIE e aproximadamente o dobro para a análise em 50\% do LIE.

\section{NOMENCLATURA}

$\begin{array}{clc}C_{P l} & \text { Calor específico à pressão constante } & \mathrm{J} / \mathrm{mol} \mathrm{K} \\ M M & \text { Massa molar do gás } & \mathrm{kg} / \mathrm{mol} \\ P_{a m b} & \text { Pressão ambiente } & \mathrm{Pa} \\ P_{a r m} & \text { Pressão no reservatório } & \mathrm{Pa} \\ R & \text { Constante dos gases } & \mathrm{J} / \mathrm{mol} \mathrm{K} \\ T & \text { Temperatura absoluta } & \mathrm{K} \\ T_{a r m} & \text { Temperatura no reservatório } & \mathrm{K} \\ T_{b} & \text { Temperatura de bolha } & \mathrm{K} \\ v & \text { Velocidade de liberação } & \mathrm{m} / \mathrm{s} \\ x_{\text {flash }}^{\text {gas }} & \text { Fração mássica vaporizada } & - \\ \Delta H_{\text {vap }} & \text { Energia de vaporização } & \mathrm{kJ} / \mathrm{mol} \\ \rho_{l} & \text { Densidade do líquido } & \mathrm{kg} / \mathrm{m}^{3} \\ \sigma & \text { Coeficiente de tensão superficial } & \mathrm{N} / \mathrm{m} \\ \gamma & \text { Constante adiabática } & -\end{array}$

\section{REFERÊNCIAS}

BOSSERT, J. A. Hazardous locations: A guide for the design, construction and installation of equipment in explosive atmospheres. Canadian Standards Association, 3 ed., Toronto, 2001 .

CALAY, R. K.; HOLDO, A. E. Modelling the dispersion of flashing jets using CFD. J. Hazard. Mat., v. 154, p. 1198-1209, 2008.

ECKHOFF, R. K. Explosion Hazards in the Process Industries. Elsevier, 2 ed., University of Bergen, Norway, 2016.

SISSOM, L. E.; PITTS, D. R. Fenômenos de transporte. Trad. De Adir M. Luiz, Rio de Janeiro: Editora Guanabara, 1988.

TNO Yellow Book. Methods for the calculation of physical effect. SDU, The Hague. 3 ed., 2 rev., 2005

ZOHDIRAD, H.; EBADI, T.; GIVEHCHI, S.; MEYSAMI, H. Grid-based individual risk calculation in the classification of hazardous area with a risk-based approach. J. Loss Prev. Proc. Ind., v. 43, p. 98-105, 2016. 